\title{
UNICITÉ FORTE À L'INFINI POUR KDV
}

\author{
LuC RoBBiano ${ }^{1}$
}

\begin{abstract}
In this paper we prove that if a solution of $\mathrm{KdV}$ equation decreases fast enough (i.e. like $\mathrm{e}^{-x^{\alpha}}$ where $\left.\alpha>9 / 4\right)$ and if the Cauchy data is null for $x$ large enough then the solution is zero. We prove a Carleman's estimate and the uniqueness result follows.
\end{abstract}

Résumé. Dans ce papier nous prouvons que si une solution de KdV est suffisamment décroissante à l'infini (c'est-à-dire comme $\mathrm{e}^{-x^{\alpha}}$ où $\alpha>9 / 4$ ) et si la donnée de Cauchy est nulle pour $x$ assez grand alors la solution est nulle. Ce résultat est la conséquence d'une inégalité de Carleman adaptée à la décroissance de la solution à l'infini.

Classification Mathématique. 35Q53, 35A07.

Reçu le 14 Janvier 2002.

\section{INTRODUCTION}

Le but de cet article est de démontrer un résultat d'unicité à l'infini pour KdV linéaire. Nous nous sommes inspirés d'un résultat de Kenig et al. [7]. Rapidement décrit, leur résultat est le suivant, soit $v$ une solution de $P v=0$ où $P$ a pour partie principale $\partial_{t}+\partial_{x}^{3}$, si $v(0, x)=0, v(1, x)=0$ et $|v(t, x)| \leqslant C \mathrm{e}^{-\beta x}$, pour tout $t \in[0,1]$ et tout $x>0$, alors $v=0$. Il est bien clair que le résultat est aussi valable si $v=0$ sur $t=t_{1}$ et $t=t_{2}$. Ici nous démontrons un résultat analogue mais en supposant seulement que $v=0$ sur $t=0$. Par contre nous faisons une hypothèse beaucoup plus forte de décroissance à l'infini, en effet nous supposons que $|v(t, x)| \leqslant C \mathrm{e}^{-x^{\alpha}}$ où $\alpha>9 / 4$.

Il faut également citer un certain nombre de résultats qui sont reliés au problème. La propriété de prolongement unique est connue pour KdV. Elle a été prouvée par Saut et Scheurer [9]. Cette propriété signifie que si $v$ est une solution de $\mathrm{KdV}$, et si $v$ s'annule sur un ouvert contenant $\left(t_{0}, x_{0}\right)$ alors $v\left(t_{0}, x\right)=0$ pour tout $x$. Une telle propriété est vraie pour des équations du type la chaleur (voir par exemple Saut et Scheurer [9], Fursikov et Imanuvilov [6]), et pour des équations du type Schrödinger (voir Lascar et Zuily [8]). Des résultats de régularité analytique sont connus pour KdV (voir Bourgain [4], Tarama [10]), qui ont pour conséquence des résultats de prolongement unique.

Mots-clés et phrases : Korteweg de Vries, unicité, inégalité de Carleman.

1 Université de Versailles-Saint Quentin, LAMA, UMR 8100 du CNRS, bâtiment Fermat, 45 avenue des États-Unis,

78035 Versailles Cedex, France ; e-mail : robbiano@math.uvsq.fr 


\section{Hypothèses et RÉSUltats}

Notons $P=\partial_{t}+\partial_{x}^{3}+\frac{a(t, x)}{x} \partial_{x}^{2}+\frac{b(t, x)}{x^{2}} \partial_{x}+\frac{c(t, x)}{x^{3}}$ où $a, b$ et $c$ sont des fonctions $L^{\infty}$. Précisément, il existe $C>0, \delta>0$ et $K>0$ telles que $\forall x>K$ et $\forall t \in[0, \delta]$ alors $|a(t, x)|+|b(t, x)|+|c(t, x)| \leqslant C$.

Théorème 2.1. Soit $v(t, x)$ une fonction $C^{1}$ en temps et $C^{3}$ en espace, on suppose qu'il existe $K>0, \delta>0$, $C>0$ et $\alpha>9 / 4$ telles que pour tout $x>K$, pour tout $t \in[0, \delta]$, alors $\sum_{j=0}^{j=3}\left|\partial_{x}^{j} v(t, x)\right|+\left|\partial_{t} v(t, x)\right| \leqslant C \mathrm{e}^{-x^{\alpha}}$. Alors, il existe $K^{\prime}>0$ telle que si $P v(t, x)=0$ et $v(0, x)=0$ pour $x>K$ et $t \in[0, \delta]$, alors $v(t, x)=0$ pour $x>K^{\prime}$ et $t \in\left[0,1 / K^{\prime}\right]$.

Ce résultat repose sur une inégalité de Carleman que nous allons décrire.

Plutôt que de travailler à l'infini nous allons nous ramener près de 0 . Notons $u(t, x)=v(t, 1 / x)$. En notant $X=1 / x$ où $X \in] K,+\infty$ [ ( $X$ c'est la variable $x$ du Th. 2.1) et $x \in] 0,1 / K\left[\right.$, on a $\partial_{X}=-x^{2} \partial_{x}$, $\partial_{X}^{2}=2 x^{3} \partial_{x}+x^{4} \partial_{x}^{2}$ et $\partial_{X}^{3}=-6 x^{4} \partial_{x}-6 x^{5} \partial_{x}^{2}-x^{6} \partial_{x}^{3}$. De sorte que l'opérateur $P$ s'écrit dans les nouvelles variables $Q=\partial_{t}-x^{6} \partial_{x}^{3}+a(t, x) x^{5} \partial_{x}^{2}+b(t, x) x^{4} \partial_{x}+c(t, x) x^{3}$. Les fonctions $a(t, x), b(t, x)$ et $c(t, x)$ se calculent à partir des coefficients $a(t, X), b(t, X)$ et $c(t, X)$ de $P$. On vérifie facilement qu'il existe $C>0, \varepsilon>0$ telles que $|a(t, x)|+|b(t, x)|+|c(t, x)| \leqslant C$ pour $x \in[0, \varepsilon]$ et $t \in[0, \delta]$.

Théorème 2.2. On suppose que $u, \partial_{x} u, \partial_{x}^{2} u, \partial_{x}^{3} u$, et $\partial_{t} u$ sont bornées. Soit $\alpha>9 / 4$, il existe $\beta>0$ tels que si $u(t, x)$ est à support dans $\left\{(t, x), t \leqslant \delta, x^{\alpha} \leqslant(\delta-t)^{\beta}, 0<x\right\}$. Si $u(0, x)=0$ pour $x<x_{0}$ où $x_{0}>0$ alors il existe $C>0, \gamma_{0}>0$ telles pour tout $\gamma \geqslant \gamma_{0}$, on a

$$
\begin{aligned}
& \gamma\left\|\mathrm{e}^{\gamma x^{-\alpha}(\delta-t)^{\beta}} x^{-\frac{\alpha}{2}+5}(\delta-t)^{\frac{\beta}{2}} \partial_{x}^{2} u\right\|^{2}+\gamma^{3}\left\|\mathrm{e}^{\gamma x^{-\alpha}(\delta-t)^{\beta}} x^{-\frac{3 \alpha}{2}+4}(\delta-t)^{\frac{3 \beta}{2}} \partial_{x} u\right\|^{2} \\
& +\gamma^{5}\left\|\mathrm{e}^{\gamma x^{-\alpha}(\delta-t)^{\beta}} x^{-\frac{5 \alpha}{2}+3}(\delta-t)^{\frac{5 \beta}{2}} u\right\|^{2}+\gamma\left\|\mathrm{e}^{\gamma x^{-\alpha}(\delta-t)^{\beta}} x^{-\frac{\alpha}{2}+\frac{5}{2}}(\delta-t)^{\frac{\beta}{2}-\frac{1}{2}} \partial_{x} u\right\|^{2} \\
& +\gamma^{3}\left\|\mathrm{e}^{\gamma x^{-\alpha}(\delta-t)^{\beta}} x^{-\frac{3 \alpha}{2}+\frac{3}{2}}(\delta-t)^{\frac{3 \beta}{2}-\frac{1}{2}} u\right\|^{2}+\gamma\left\|\mathrm{e}^{\gamma x^{-\alpha}(\delta-t)^{\beta}} x^{-\frac{\alpha}{2}}(\delta-t)^{\frac{\beta}{2}-1} u\right\|^{2} \\
\leqslant & C\left\|\mathrm{e}^{\gamma x^{-\alpha}(\delta-t)^{\beta}} Q u\right\|^{2} .
\end{aligned}
$$

Dans ce théorème $u$ est à support compact. Toutes les expressions sont donc finies. Si $u$ et ses dérivées sont suffisamment décroissantes en $x=0(2.1)$ sera encore vérifiée par $u$. Cette remarque sera précisée dans la preuve du théorème 2.1 .

Commentaires. Le théorème 2.1 et le résultat de Saut et Scheurer [9] (unicité à partir d'un ouvert) permettent d'obtenir un résultat d'unicité globale pour $t \in[0, \delta]$ et $x \in \mathbb{R}$.

L'hypothèse d'annulation est très forte mais si nous notons

$$
\begin{aligned}
& a=i\left(\tau+x^{6} \xi^{3}-3 \alpha^{2} x^{-2 \alpha+4}(\delta-t)^{2 \beta} \gamma^{2} \xi-6 x^{4} \xi\right) \\
& b=\beta x^{-\alpha}(\delta-t)^{\beta-1} \gamma+3 \alpha x^{-\alpha+5}(\delta-t)^{\beta} \gamma \xi^{2}-\alpha^{3} x^{-3 \alpha+3}(\delta-t)^{3 \beta} \gamma^{3}+6 x^{5} \xi^{2}
\end{aligned}
$$

qui sont les symboles principaux des opérateurs $A$ et $B$ définis ci-dessous, le calcul du crochets de Poisson $\frac{1}{i}\{b, a\}$ sur $b=0$ est égal à

$$
\gamma x^{-\alpha}(\delta-t)^{\beta}\left(144 \alpha(\alpha-1) x^{10} \xi^{4}+12 \beta(5 \alpha-6) x^{5}(\delta-t)^{-1} \xi^{2}+\frac{\beta}{\alpha}(4 \alpha \beta-9 \beta-\alpha)(\delta-t)^{-2}\right) .
$$

Ceci n'est positif que pour $\alpha>9 / 4$. Si $\alpha \leqslant 9 / 4$ ce crochet peut être strictement négatif et généralement dans ce cas on ne peut pas prouver l'unicité et dans de nombreux cas on peut construire des contre-exemples (voir par exemple Alinhac [1], Alinhac et Baouendi [2]). Néanmoins cette condition de décroissance n'est justifiée que par des raisons techniques, dont la pertinence est un problème ouvert. 
Si on se place dans le cadre Kenig et al. [7], c'est-à-dire si on suppose de plus que $v(1, x)=0$ pour $x>K$, alors on peut utiliser un poids avec $\beta=0$. On voit que le crochet ci-dessus est strictement positif dès que $\alpha>1$ et identiquement nul si $\alpha=1$. Il semble donc que si la technique d'inégalité de Carleman en norme $L^{2}$ utilisée ici ne donne pas les résultats optimaux, elle permet de s'en approcher. Kenig et al. [7] utilisent des inégalités de Carleman dans $L^{p}$. Cette technique donne des résultats plus fin mais est beaucoup plus difficile à mettre en œuvre. La technique $L^{2}$ nous permet de prendre des poids qui dépendent de $t$. Il n'est pas clair qu'on puisse prendre de tels poids dans la technique $L^{p}$.

Le poids utilisé est à rapprocher des techniques d'éclatement de Baouendi et Zachmanoglou [3] qui consiste à poser $X=\frac{x}{(\delta-t) t}$ et ensuite de prendre un poids $\mathrm{e}^{\gamma X^{-\alpha}}$, ce qui revient à prendre un poids de la forme $\mathrm{e}^{\gamma x^{-\alpha}(\delta-t)^{\alpha} t^{\alpha}}$. Ici nous n'éclatons que du côté $t=\delta$. Il faut remarquer que si nous essayons d'éclater du côté $t=0$ ou des deux côtés, le crochet n'est alors jamais positif même en prenant $\alpha$ très grand. Ces calculs ne donnant pas de résultats montrent d'une part, qu'il ne faut pas croire qu'il suffit de prendre des données à décroissance très rapide à l'infini pour obtenir un résultat, d'autre part que le sens du temps joue un rôle dans ce problème.

Dans la preuve nous n'utilisons pas le calcul symbolique. Le calcul du crochet de $a$ et de $b$ est plus aisé que les intégrations par parties faites plus bas mais d'une part nous ne savons pas dans quelle classe de symboles il faut se placer pour que le calcul symbolique soit correct, et d'autre part, nous aurions besoin d'une inégalité de Gårding, qui n'est pas simple à démontrer dans des classes analogues à celles de Colombini et al. [5]. Bien sûr, nous pensons que de telles classes de symboles seraient nécessaires ici. Le calcul fait plus bas est peut être pénible mais complètement élémentaire. Les étapes en sont claires et suivent celles des preuves par calcul symbolique. Premièrement, on calcule le crochet $[A, B]$ ou d'une façon analogue, on intègre par partie les termes de $2 \operatorname{Re}(A u, B u)$, deuxièmement, on se place sur $a=0$ et $b=0$, c'est-à-dire, on élimine les termes en $\partial_{t} u$ grâce à $A$ et $\gamma^{3} u$ grâce à $B$, ce qui permet d'obtenir une expression dont le symbole est celui de la formule (2.2).

\section{DÉmonstrations}

Commençons par prouver le théorème 2.2. Nous pouvons supposer que $Q$ est égale à $\partial_{t}-x^{6} \partial_{x}^{3}$. En effet les termes d'ordre inférieurs sont facilement majorés par

$$
\begin{aligned}
C\left\|\mathrm{e}^{\gamma x^{-\alpha}(\delta-t)^{\beta}} x^{-\frac{\alpha}{2}+5}(\delta-t)^{\frac{\beta}{2}} \partial_{x}^{2} u\right\|^{2} & +C\left\|\mathrm{e}^{\gamma x^{-\alpha}(\delta-t)^{\beta}} x^{-\frac{3 \alpha}{2}+4}(\delta-t)^{\frac{3 \beta}{2}} \partial_{x} u\right\|^{2} \\
& +C\left\|\mathrm{e}^{\gamma x^{-\alpha}(\delta-t)^{\beta}} x^{-\frac{5 \alpha}{2}+3}(\delta-t)^{\frac{5 \beta}{2}} u\right\|^{2}
\end{aligned}
$$

pour une constante $C$ assez grande. Nous utilisons ici ainsi que dans la suite que, sur le support de $u$, $1 \leqslant x^{-\alpha}(\delta-t)^{\beta}$. Par exemple, nous avons que

$$
\left\|\mathrm{e}^{\gamma x^{-\alpha}(\delta-t)^{\beta}} x^{5} \partial_{x}^{2} u\right\|^{2} \leqslant\left\|\mathrm{e}^{\gamma x^{-\alpha}(\delta-t)^{\beta}} x^{-\frac{\alpha}{2}+5}(\delta-t)^{\frac{\beta}{2}} \partial_{x}^{2} u\right\|^{2} .
$$

On peut alors absorber ce terme par le côté gauche de $(2.1)$. Nous conjuguons $Q$ avec le poids $\mathrm{e}^{\gamma x^{-\alpha}(\delta-t)^{\beta}}$, et nous noterons

$$
Q_{\gamma}=\mathrm{e}^{\gamma x^{-\alpha}(\delta-t)^{\beta}} Q \mathrm{e}^{-\gamma x^{-\alpha}(\delta-t)^{\beta}}
$$

on obtient que $Q_{\gamma}=A+B+R_{1}$, avec

$$
\begin{aligned}
& A=\partial_{t}-x^{6} \partial_{x}^{3}-3 \alpha^{2} x^{-2 \alpha+4}(\delta-t)^{2 \beta} \gamma^{2} \partial_{x} \\
& B=\beta x^{-\alpha}(\delta-t)^{\beta-1} \gamma-3 \alpha x^{-\alpha+5}(\delta-t)^{\beta} \gamma \partial_{x}^{2}-\alpha^{3} x^{-3 \alpha+3}(\delta-t)^{3 \beta} \gamma^{3}
\end{aligned}
$$


L'inégalité (2.1) est donc équivalente à la suivante,

$$
\begin{aligned}
\gamma\left\|x^{-\frac{\alpha}{2}+5}(\delta-t)^{\frac{\beta}{2}} \partial_{x}^{2} u\right\|^{2} & +\gamma^{3}\left\|x^{-\frac{3 \alpha}{2}+4}(\delta-t)^{\frac{3 \beta}{2}} \partial_{x} u\right\|^{2} \\
& +\gamma^{5}\left\|x^{-\frac{5 \alpha}{2}+3}(\delta-t)^{\frac{5 \beta}{2}} u\right\|^{2}+\gamma\left\|x^{-\frac{\alpha}{2}+\frac{5}{2}}(\delta-t)^{\frac{\beta}{2}-\frac{1}{2}} \partial_{x} u\right\|^{2} \\
& +\gamma^{3}\left\|x^{-\frac{3 \alpha}{2}+\frac{3}{2}}(\delta-t)^{\frac{3 \beta}{2}-\frac{1}{2}} u\right\|^{2}+\gamma\left\|x^{-\frac{\alpha}{2}}(\delta-t)^{\frac{\beta}{2}-1} u\right\|^{2} \\
\leqslant & C\left\|Q_{\gamma} u\right\|^{2} .
\end{aligned}
$$

En effet, il suffit de remplacer dans (3.1) $u$ par $\mathrm{e}^{\gamma x^{-\alpha}(\delta-t)^{\beta}} u$ pour obtenir (2.1). Le terme $R_{1} u$ est un reste car $\left\|R_{1} u\right\|^{2}$ est majoré par $\frac{C}{\gamma}$ fois le terme de gauche de (3.1). Il est aussi facile de vérifier que le terme de gauche de (3.1) est équivalent au terme de gauche de (2.1).

Nous avons

$$
\|(A+B) u\|^{2}=\|A u\|^{2}+\|B u\|^{2}+2 \operatorname{Re}(A u, B u) .
$$

Calculons un à un les termes de $2 \operatorname{Re}(A u, B u)$.

Nous noterons $R$ un terme majoré par

$$
\begin{aligned}
\varepsilon\|A u\|^{2}+\varepsilon\|B u\|^{2} & +C_{\varepsilon}\left\|x^{-\frac{\alpha}{2}+5}(\delta-t)^{\frac{\beta}{2}} \partial_{x}^{2} u\right\|^{2}+C_{\varepsilon} \gamma^{2}\left\|x^{-\frac{3 \alpha}{2}+4}(\delta-t)^{\frac{3 \beta}{2}} \partial_{x} u\right\|^{2} \\
& +C_{\varepsilon} \gamma^{4}\left\|x^{-\frac{5 \alpha}{2}+3}(\delta-t)^{\frac{5 \beta}{2}} u\right\|^{2}+C_{\varepsilon}\left\|x^{-\frac{\alpha}{2}+\frac{5}{2}}(\delta-t)^{\frac{\beta}{2}-\frac{1}{2}} \partial_{x} u\right\|^{2} \\
& +C_{\varepsilon} \gamma^{2}\left\|x^{-\frac{3 \alpha}{2}+\frac{3}{2}}(\delta-t)^{\frac{3 \beta}{2}-\frac{1}{2}} u\right\|^{2}+C_{\varepsilon}\left\|x^{-\frac{\alpha}{2}}(\delta-t)^{\frac{\beta}{2}-1} u\right\|^{2} \\
\left(C_{1}\right)= & 2 \operatorname{Re}\left(\partial_{t} u, \beta x^{-\alpha}(\delta-t)^{\beta-1} \gamma u\right)=(\beta-1) \beta \gamma\left(u, x^{-\alpha}(\delta-t)^{\beta-2} u\right) \\
\left(C_{2}\right)= & 2 \operatorname{Re}\left(\partial_{t} u,-3 \alpha x^{-\alpha+5}(\delta-t)^{\beta} \gamma \partial_{x}^{2} u\right) \\
= & 2 \operatorname{Re}\left(\partial_{t} \partial_{x} u, 3 \alpha x^{-\alpha+5}(\delta-t)^{\beta} \gamma \partial_{x} u\right)+2 \operatorname{Re}\left(\partial_{t} u, 3 \alpha(-\alpha+5) x^{-\alpha+4}(\delta-t)^{\beta} \gamma \partial_{x} u\right) \\
= & \left(\partial_{x} u, 3 \alpha \beta x^{-\alpha+5}(\delta-t)^{\beta-1} \gamma \partial_{x} u\right) \\
& +2 \operatorname{Re}\left(A u+x^{6} \partial_{x}^{3} u+3 \alpha^{2} x^{-2 \alpha+4}(\delta-t)^{2 \beta} \gamma^{2} \partial_{x} u, 3 \alpha(-\alpha+5) x^{-\alpha+4}(\delta-t)^{\beta} \gamma \partial_{x} u\right) .
\end{aligned}
$$

Comme $\left|\operatorname{Re}\left(A u, x^{-\alpha+4}(\delta-t)^{\beta} \gamma \partial_{x} u\right)\right| \leqslant \varepsilon\|A u\|^{2}+C_{\varepsilon} \gamma^{2}\left\|x^{-\frac{3 \alpha}{2}+4}(\delta-t)^{\frac{3 \beta}{2}} \partial_{x} u\right\|^{2} \leqslant R$, on obtient que

$$
\begin{aligned}
\left(C_{2}\right)= & 6 \alpha(\alpha-5) \gamma \operatorname{Re}\left(\partial_{x}^{2} u, x^{-\alpha+10}(\delta-t)^{\beta} \partial_{x}^{2} u\right) \\
& +18 \alpha^{3}(\alpha-5) \gamma^{3} \operatorname{Re}\left(u, x^{-3 \alpha+8}(\delta-t)^{3 \beta} \partial_{x}^{2} u\right) \\
& +3 \alpha \beta \gamma\left(\partial_{x} u, x^{-\alpha+5}(\delta-t)^{\beta-1} \partial_{x} u\right)+R
\end{aligned}
$$

car

$$
\left|\left(\partial_{x}^{2} u, x^{-\alpha+9}(\delta-t)^{\beta} \gamma \partial_{x} u\right)\right| \leqslant\left\|x^{-\frac{\alpha}{2}+5}(\delta-t)^{\frac{\beta}{2}} \partial_{x}^{2} u\right\|^{2}+\gamma^{2}\left\|x^{-\frac{3 \alpha}{2}+4}(\delta-t)^{\frac{3 \beta}{2}} \partial_{x} u\right\|^{2} \leqslant R
$$

et

$$
\begin{aligned}
& \left|\left(u, x^{-3 \alpha+7}(\delta-t)^{3 \beta} \gamma^{3} \partial_{x} u\right)\right| \leqslant \gamma^{2}\left\|x^{-\frac{3 \alpha}{2}+4}(\delta-t)^{\frac{3 \beta}{2}} \partial_{x} u\right\|^{2}+\gamma^{4}\left\|x^{-\frac{5 \alpha}{2}+3}(\delta-t)^{\frac{5 \beta}{2}} u\right\|^{2} \leqslant R \\
& \left(C_{3}\right)=2 \operatorname{Re}\left(\partial_{t} u,-\alpha^{3} x^{-3 \alpha+3}(\delta-t)^{3 \beta} \gamma^{3} u\right)=-3 \alpha^{3} \beta \gamma^{3}\left(u, x^{-3 \alpha+3}(\delta-t)^{3 \beta-1} u\right) \\
& \left(C_{4}\right)=2 \operatorname{Re}\left(-x^{6} \partial_{x}^{3} u, \beta x^{-\alpha}(\delta-t)^{\beta-1} \gamma u\right) \\
& \quad=2 \operatorname{Re}\left(\partial_{x}^{2} u, \beta x^{-\alpha+6}(\delta-t)^{\beta-1} \gamma \partial_{x} u\right)+2 \operatorname{Re}\left(\partial_{x}^{2} u, \beta(-\alpha+6) x^{-\alpha+5}(\delta-t)^{\beta-1} \gamma u\right) \\
& \quad=3 \beta(\alpha-6) \gamma\left(\partial_{x} u, x^{-\alpha+5}(\delta-t)^{\beta-1} \partial_{x} u\right)+R
\end{aligned}
$$


car

$$
\begin{aligned}
&\left|\left(\partial_{x} u, x^{-\alpha+4}(\delta-t)^{\beta-1} \gamma u\right)\right| \leqslant\left\|x^{-\frac{\alpha}{2}+\frac{5}{2}}(\delta-t)^{\frac{\beta}{2}-\frac{1}{2}} \partial_{x} u\right\|^{2}+\gamma^{2}\left\|x^{-\frac{3 \alpha}{2}+\frac{3}{2}}(\delta-t)^{\frac{3 \beta}{2}-\frac{1}{2}} u\right\|^{2} \leqslant R \\
&\left(C_{5}\right)= 2 \operatorname{Re}\left(-x^{6} \partial_{x}^{3} u,-3 \alpha x^{-\alpha+5}(\delta-t)^{\beta} \gamma \partial_{x}^{2} u\right) \\
&= 3 \alpha(\alpha-11) \gamma\left(\partial_{x}^{2} u, x^{-\alpha+10}(\delta-t)^{\beta} \partial_{x}^{2} u\right) \\
&\left(C_{6}\right)= 2 \operatorname{Re}\left(-x^{6} \partial_{x}^{3} u,-\alpha^{3} x^{-3 \alpha+3}(\delta-t)^{3 \beta} \gamma^{3} u\right) \\
&= 2 \operatorname{Re}\left(\partial_{x}^{2} u,-\alpha^{3} x^{-3 \alpha+9}(\delta-t)^{3 \beta} \gamma^{3} \partial_{x} u\right) \\
&+2 \operatorname{Re}\left(\partial_{x}^{2} u,-(-3 \alpha+9) \alpha^{3} x^{-3 \alpha+8}(\delta-t)^{3 \beta} \gamma^{3} u\right) \\
&=\left(\partial_{x} u, \alpha^{3}(-3 \alpha+9) x^{-3 \alpha+8}(\delta-t)^{3 \beta} \gamma^{3} \partial_{x} u\right) \\
&+2 \operatorname{Re}\left(\partial_{x}^{2} u,-(-3 \alpha+9) \alpha^{3} x^{-3 \alpha+8}(\delta-t)^{3 \beta} \gamma^{3} u\right) \\
&= 3(3 \alpha-9) \alpha^{3} \gamma^{3} \operatorname{Re}\left(u, x^{-3 \alpha+8}(\delta-t)^{3 \beta} \partial_{x}^{2} u\right)+R
\end{aligned}
$$

en utilisant la majoration (3.4).

$$
\begin{aligned}
\left(C_{7}\right) & =2 \operatorname{Re}\left(-3 \alpha^{2} x^{-2 \alpha+4}(\delta-t)^{2 \beta} \gamma^{2} \partial_{x} u, \beta x^{-\alpha}(\delta-t)^{\beta-1} \gamma u\right) \\
& =3 \alpha^{2}(-3 \alpha+4) \beta \gamma^{3}\left(u, x^{-3 \alpha+3}(\delta-t)^{3 \beta-1} u\right) \\
\left(C_{8}\right) & =2 \operatorname{Re}\left(-3 \alpha^{2} x^{-2 \alpha+4}(\delta-t)^{2 \beta} \gamma^{2} \partial_{x} u,-3 \alpha x^{-\alpha+5}(\delta-t)^{\beta} \gamma \partial_{x}^{2} u\right) \\
& =-9 \alpha^{3}(-3 \alpha+9) \gamma^{3}\left(\partial_{x} u, x^{-3 \alpha+8}(\delta-t)^{3 \beta} \partial_{x} u\right) \\
& =9 \alpha^{3}(-3 \alpha+9) \gamma^{3}\left(u, x^{-3 \alpha+8}(\delta-t)^{3 \beta} \partial_{x}^{2} u\right)+R
\end{aligned}
$$

en utilisant la majoration (3.4).

$$
\begin{aligned}
\left(C_{9}\right) & =2 \operatorname{Re}\left(-3 \alpha^{2} x^{-2 \alpha+4}(\delta-t)^{2 \beta} \gamma^{2} \partial_{x} u,-\alpha^{3} x^{-3 \alpha+3}(\delta-t)^{3 \beta} \gamma^{3} u\right) \\
& =3 \alpha^{5}(5 \alpha-7) \gamma^{5}\left(u, x^{-5 \alpha+6}(\delta-t)^{5 \beta} u\right) .
\end{aligned}
$$

En regroupant les termes de $\left(C_{1}\right)$ à $\left(C_{9}\right)$, on obtient que,

$$
\begin{aligned}
2 \operatorname{Re}(A u, B u)= & 9 \alpha(\alpha-7) \gamma\left(\partial_{x}^{2} u, x^{-\alpha+10}(\delta-t)^{\beta} \partial_{x}^{2} u\right) \\
& -36 \alpha^{3} \gamma^{3}\left(u, x^{-3 \alpha+8}(\delta-t)^{3 \beta} \partial_{x}^{2} u\right) \\
& +3 \alpha^{5}(5 \alpha-7) \gamma^{5}\left(u, x^{-5 \alpha+6}(\delta-t)^{5 \beta} u\right) \\
& +6(\alpha-3) \beta \gamma\left(\partial_{x} u, x^{-\alpha+5}(\delta-t)^{\beta-1} \partial_{x} u\right) \\
& -12 \alpha^{2}(\alpha-1) \beta \gamma^{3}\left(u, x^{-3 \alpha+3}(\delta-t)^{3 \beta-1} u\right) \\
& +\beta(\beta-1) \gamma\left(u, x^{-\alpha}(\delta-t)^{\beta-2} u\right)+R .
\end{aligned}
$$

Nous allons maintenant utiliser l'égalité

$$
\alpha^{3} x^{-3 \alpha+3}(\delta-t)^{3 \beta} \gamma^{3} u=-B u+\beta x^{-\alpha}(\delta-t)^{\beta-1} \gamma u-3 \alpha x^{-\alpha+5}(\delta-t)^{\beta} \gamma \partial_{x}^{2} u
$$

pour démontrer que les deuxième, troisième et cinquième termes de (3.6) s'écrivent en fonction des trois autres et de $R$. On a,

$$
\begin{aligned}
-36 \alpha^{3} \gamma^{3}\left(u, x^{-3 \alpha+8}(\delta-t)^{3 \beta} \partial_{x}^{2} u\right) & =-36\left(-B u+\beta x^{-\alpha}(\delta-t)^{\beta-1} \gamma u-3 \alpha x^{-\alpha+5}(\delta-t)^{\beta} \gamma \partial_{x}^{2} u, x^{5} \partial_{x}^{2} u\right) \\
& =36 \beta \gamma\left(\partial_{x} u, x^{-\alpha+5}(\delta-t)^{\beta-1} \partial_{x} u\right)+108 \alpha \gamma\left(\partial_{x}^{2} u, x^{-\alpha+10}(\delta-t)^{\beta} \partial_{x}^{2} u\right)+R
\end{aligned}
$$


$\left.\operatorname{car} \mid B u, x^{5} \partial_{x}^{2} u\right) \mid \leqslant \varepsilon\|B u\|+C_{\varepsilon}\left\|x^{-\frac{\alpha}{2}+5}(\delta-t)^{\frac{\beta}{2}} \partial_{x}^{2} u\right\|^{2} \leqslant R$ et $\left|\gamma\left(u, x^{-\alpha+4}(\delta-t)^{\beta-1} \partial_{x} u\right)\right| \leqslant\left\|x^{-\frac{\alpha}{2}}(\delta-t)^{\frac{\beta}{2}-1} u\right\|^{2}$ $+\gamma^{2}\left\|x^{-\frac{3 \alpha}{2}+4}(\delta-t)^{\frac{3 \beta}{2}} \partial_{x} u\right\|^{2} \leqslant R$.

Notons $S u=-B u+\beta x^{-\alpha}(\delta-t)^{\beta-1} \gamma u-3 \alpha x^{-\alpha+5}(\delta-t)^{\beta} \gamma \partial_{x}^{2} u$, on a

$$
\begin{aligned}
3 \alpha^{5}(5 \alpha-7) \gamma^{5}\left(u, x^{-5 \alpha+6}(\delta-t)^{5 \beta} u\right)= & \frac{3}{\alpha \gamma}(5 \alpha-7)\left(S u, x^{\alpha}(\delta-t)^{-\beta} S u\right) \\
= & 27 \alpha(5 \alpha-7) \gamma\left(\partial_{x}^{2} u, x^{-\alpha+10}(\delta-t)^{\beta} \partial_{x}^{2} u\right) \\
& +18(5 \alpha-7) \beta \gamma\left(\partial_{x} u, x^{-\alpha+5}(\delta-t)^{\beta-1} \partial_{x} u\right) \\
& +\frac{3}{\alpha}(5 \alpha-7) \beta^{2} \gamma\left(u, x^{-\alpha}(\delta-t)^{\beta-2} u\right)+R
\end{aligned}
$$

$\operatorname{car} \frac{1}{\gamma}\left|\left(B u, x^{\alpha}(\delta-t)^{-\beta} B u\right)\right| \leqslant \varepsilon\|B u\|^{2} \leqslant R$ et $\left|\frac{1}{\gamma}\left(B u, \beta(\delta-t)^{-1} \gamma u-3 \alpha x^{5} \gamma \partial_{x}^{2} u\right)\right| \leqslant \varepsilon\|B u\|^{2}+C_{\varepsilon} \| x^{-\frac{\alpha}{2}}(\delta-$ $t)^{\frac{\beta}{2}-1} u\left\|^{2}+C_{\varepsilon}\right\| x^{-\frac{\alpha}{2}+5}(\delta-t)^{\frac{\beta}{2}} \partial_{x}^{2} u \|^{2} \leqslant R$.

$$
\begin{aligned}
-12 \alpha^{2}(\alpha-1) \beta \gamma^{3}\left(u, x^{-3 \alpha+3}(\delta-t)^{3 \beta-1} u\right)= & -12 \frac{\beta(\alpha-1)}{\alpha}\left(S u,(\delta-t)^{-1} u\right) \\
= & -36 \beta(\alpha-1) \gamma\left(\partial_{x} u, x^{-\alpha+5}(\delta-t)^{\beta-1} \partial_{x} u\right) \\
& -12 \frac{\beta^{2}(\alpha-1)}{\alpha} \gamma\left(u, x^{-\alpha}(\delta-t)^{\beta-2} u\right)+R
\end{aligned}
$$

d'après (3.5) et l'inégalité $\left|\left(B u,(\delta-t)^{-1} u\right)\right| \leqslant \varepsilon\|B u\|+C_{\varepsilon}\left\|x^{-\frac{\alpha}{2}}(\delta-t)^{\frac{\beta}{2}-1} u\right\|^{2} \leqslant R$.

En utilisant les calculs (3.7-3.9) dans le résultat (3.6), on obtient,

$$
\begin{aligned}
2 \operatorname{Re}(A u, B u)= & 144 \alpha(\alpha-1) \gamma\left(\partial_{x}^{2} u, x^{-\alpha+10}(\delta-t)^{\beta} \partial_{x}^{2} u\right) \\
& +12(5 \alpha-6) \beta \gamma\left(\partial_{x} u, x^{-\alpha+5}(\delta-t)^{\beta-1} \partial_{x} u\right) \\
& +\frac{\beta}{\alpha}(4 \alpha \beta-9 \beta-\alpha) \gamma\left(u, x^{-\alpha}(\delta-t)^{\beta-2} u\right)+R .
\end{aligned}
$$

Si $\alpha>9 / 4$, on peut choisir $\beta$ assez grand pour que le terme de droite de (3.10) soit strictement positif modulo $R$. Les calculs $(3.7,3.8)$ et $(3.9)$ permettent de montrer qu'il existe $C_{0}>0$ et $C_{1}>0$ telles que

$$
\begin{aligned}
& C_{0}\left(\gamma^{3}\left\|x^{-\frac{3 \alpha}{2}+4}(\delta-t)^{\frac{3 \beta}{2}} \partial_{x} u\right\|^{2}+\gamma^{5}\left\|x^{-\frac{5 \alpha}{2}+3}(\delta-t)^{\frac{5 \beta}{2}} u\right\|^{2}+\gamma^{3}\left\|x^{-\frac{3 \alpha}{2}+\frac{3}{2}}(\delta-t)^{\frac{3 \beta}{2}-\frac{1}{2}} u\right\|^{2}\right) \\
\leqslant & C_{1}\left(\gamma\left\|x^{-\frac{\alpha}{2}+5}(\delta-t)^{\frac{\beta}{2}} \partial_{x}^{2} u\right\|^{2}+\gamma\left\|x^{-\frac{\alpha}{2}+\frac{5}{2}}(\delta-t)^{\frac{\beta}{2}-\frac{1}{2}} \partial_{x} u\right\|^{2}\right. \\
& \left.+\gamma\left\|x^{-\frac{\alpha}{2}}(\delta-t)^{\frac{\beta}{2}-1} u\right\|^{2}\right)+R \\
\leqslant & 2 \operatorname{Re}(A u, B u)+R .
\end{aligned}
$$

En choisissant $\varepsilon$ assez petit dans tous les calculs précédents, il existe une constante $C>0$ telle que le terme $R$ de (3.11) est majoré par

$$
\begin{aligned}
\frac{1}{2}\|A u\|^{2} & +\frac{1}{2}\|B u\|^{2}+C\left\|x^{-\frac{\alpha}{2}+5}(\delta-t)^{\frac{\beta}{2}} \partial_{x}^{2} u\right\|^{2}+C \gamma^{2}\left\|x^{-\frac{3 \alpha}{2}+4}(\delta-t)^{\frac{3 \beta}{2}} \partial_{x} u\right\|^{2} \\
& +C \gamma^{4}\left\|x^{-\frac{5 \alpha}{2}+3}(\delta-t)^{\frac{5 \beta}{2}} u\right\|^{2}+C\left\|x^{-\frac{\alpha}{2}+\frac{5}{2}}(\delta-t)^{\frac{\beta}{2}-\frac{1}{2}} \partial_{x} u\right\|^{2} \\
& +C \gamma^{2}\left\|x^{-\frac{3 \alpha}{2}+\frac{3}{2}}(\delta-t)^{\frac{3 \beta}{2}-\frac{1}{2}} u\right\|^{2}+C\left\|x^{-\frac{\alpha}{2}}(\delta-t)^{\frac{\beta}{2}-1} u\right\|^{2} .
\end{aligned}
$$


Donc en notant $D$ le terme de droite de (3.1), nous avons d'après le calcul (3.2), la majoration (3.11) et pour une constante $C_{2}>0$, l'inégalité,

$$
\|(A+B) u\|^{2} \geqslant\|A u\|^{2}+\|B u\|^{2}+C_{2} D-\frac{1}{2}\|A u\|^{2}-\frac{1}{2}\|B u\|^{2}-\frac{C}{\gamma} D
$$

ce qui permet de déduire l'inégalité (3.1) en prenant $\gamma$ assez grand.

La preuve du théorème 2.1 à partir de l'inégalité (2.1) est usuelle.

Tout d'abord, si $u$ est à support dans $\left\{(t, x), 0 \leqslant x, x^{\alpha} \leqslant(\delta-t)^{\beta}\right\}$ et si $u$ vérifie $\sum_{j=0}^{j=3}\left|\partial_{x}^{j} u(t, x)\right|+\left|\partial_{t} u(t, x)\right|$ $\leqslant C \mathrm{e}^{-x^{-\alpha^{\prime}}}$ où $9 / 4<\alpha<\alpha^{\prime}$ ( $\alpha^{\prime}$ est le $\alpha$ du Th. 2.1) alors l'inégalité (2.1) est vérifiée pour $u$. Il suffit d'appliquer $(2.1)$ à $w(t, x)=\chi_{0}\left(\varepsilon^{-1} x\right) u(t, x)$ où $\chi_{0}(x)=0$ pour $x<1 / 2$ et $\chi_{0}(x)=1$ pour $x>1$, et de faire tendre $\varepsilon$ vers 0 , pour $\gamma$ fixé.

Soit $\chi=1-\chi_{0}$ posons $u(t, x)=\chi\left(x^{\alpha}(\delta-t)^{-\beta}\right) v(t, x)$ où $v$ est la solution de KdV vérifiant les hypothèses $\mathrm{du}$ théorème 2.1, après le changement de $x$ en $1 / x$. Nous avons donc $Q v=0$ et nous pouvons appliquer l'inégalité (2.1) à $u$ grâce à la remarque ci-dessus. Nous noterons d'une part $K_{1}=\{(t, x), 0 \leqslant t \leqslant \delta / 2$, $\left.0 \leqslant x, x^{\alpha}(\delta-t)^{-\beta} \leqslant 1 / 10\right\}$ et d'autre part $K_{2}=\left\{(t, x), 0 \leqslant t \leqslant \delta, 0 \leqslant x, 1 / 2 \leqslant x^{\alpha}(\delta-t)^{-\beta} \leqslant 1\right\}$. Remarquons que sur $K_{1}$, pour tout $\varepsilon>0$ il existe $C>0$ telle que $x^{\alpha} \geqslant(\delta / 2)^{\beta} x^{\alpha}(\delta-t)^{-\beta} \geqslant C \mathrm{e}^{-\varepsilon x^{-\alpha}(\delta-t)^{\beta}}$. Dans la suite $C$ est une constante strictement positive qui change d'une ligne à l'autre. Nous pouvons donc minorer le terme de gauche de (2.1) par $C \mathrm{e}^{(10-\varepsilon) \gamma}\|v\|_{K_{1}}^{2}$ et nous pouvons majorer le terme de droite de (2.1) $\operatorname{par} C \mathrm{e}^{2 \gamma} \sum_{j=0}^{2}\left\|\partial_{x}^{j} v\right\|_{K_{2}}^{2}$. Nous avons noté $\|\cdot\|_{K_{j}}$ la norme $L^{2}$ restreinte à $K_{j}$. En faisant tendre $\gamma$ vers $+\infty$, on doit avoir $v=0$ sur $K_{1}$.

\section{RÉFÉRENCES}

[1] S. Alinhac, Non-unicité du problème de Cauchy. Ann. of Math. 117 (1983) 77-108.

[2] S. Alinhac et M.S. Baouendi, A nonuniqueness result for operators of principal type. Math. Z. 220 (1995) $561-568$.

[3] M.S. Baouendi et M.S. Zachmanoglou, Unique continuation of solutions of partial differential equations and inequalities from manifolds of any dimension. Duke Math. J. 45 (1978) 1-13.

[4] J. Bourgain, On the compactness of the support of solutions of dispersive equations. Internat. Math. Res. Notices 9 (1997) 437-447.

[5] F. Colombini, D. Del Santo et C. Zuily, The Fefferman-Phong inequality in the locally temperate Weyl calculus. Osaka J. Math. 33 (1996) 847-861.

[6] A.V. Fursikov et O.Yu. Imanuvilov, Controllability of evolution equations. Lecture Notes Ser. 34. Seoul National University, Research Institute of Mathematics. Global Analysis Research Center, Seoul (1996).

[7] C. Kenig, G. Ponce et L. Vega, On the support of solutions to the generalized KdV equation. Ann. Inst. H. Poincaré 19 (2002) 191-208.

[8] R. Lascar et C. Zuily, Unicité et non unicité du problème de Cauchy pour une classe d'opérateurs différentiels à caractéristiques doubles. Duke Math. J. 49 (1982) 137-162.

[9] J.C. Saut et B. Scheurer, Unique continuation for some evolution equations. J. Differential Equations 66 (1987) $118-139$.

[10] S. Tarama, Analytic solutions of Korteweg-de Vries equation (prépublication). 